\title{
Millennium development health metrics: where do Africa's children and women of childbearing age live?
}

\author{
Andrew J Tatem ${ }^{1,2^{*}}$, Andres J Garcia ${ }^{3,4}$, Robert W Snow ${ }^{5,6}$, Abdisalan M Noor ${ }^{5,6}$, Andrea E Gaughan ${ }^{3,4}$, \\ Marius Gilbert ${ }^{7,8}$ and Catherine Linard ${ }^{7,8}$
}

\begin{abstract}
The Millennium Development Goals (MDGs) have prompted an expansion in approaches to deriving health metrics to measure progress toward their achievement. Accurate measurements should take into account the high degrees of spatial heterogeneity in health risks across countries, and this has prompted the development of sophisticated cartographic techniques for mapping and modeling risks. Conversion of these risks to relevant population-based metrics requires equally detailed information on the spatial distribution and attributes of the denominator populations. However, spatial information on age and sex composition over large areas is lacking, prompting many influential studies that have rigorously accounted for health risk heterogeneities to overlook the substantial demographic variations that exist subnationally and merely apply national-level adjustments.

Here we outline the development of high resolution age- and sex-structured spatial population datasets for Africa in 2000-2015 built from over a million measurements from more than 20,000 subnational units, increasing input data detail from previous studies by over 400 -fold. We analyze the large spatial variations seen within countries and across the continent for key MDG indicator groups, focusing on children under 5 and women of childbearing age, and find that substantial differences in health and development indicators can result through using only national level statistics, compared to accounting for subnational variation.

Progress toward meeting the MDGs will be measured through national-level indicators that mask substantial inequalities and heterogeneities across nations. Cartographic approaches are providing opportunities for quantitative assessments of these inequalities and the targeting of interventions, but demographic spatial datasets to support such efforts remain reliant on coarse and outdated input data for accurately locating risk groups. We have shown here that sufficient data exist to map the distribution of key vulnerable groups, and that doing so has substantial impacts on derived metrics through accounting for spatial demographic heterogeneities that exist within nations across Africa.
\end{abstract}

Keywords: Population, Demography, Mapping, Millenium development goals

\section{Introduction}

The Millennium Development Goals (MDGs) were initiated to encourage development by improving social and economic conditions in the world's poorest countries [1]. In order to achieve this on a 15-year timeline, targets and indicators for poverty reduction and health improvement

\footnotetext{
* Correspondence: Andy.Tatem@gmail.com

'Department of Geography and Environment, University of Southampton, Highfield, Southampton, UK

${ }^{2}$ Fogarty International Center, National Institutes of Health, Bethesda, MD 20892, USA

Full list of author information is available at the end of the article
}

were set. There are eight goals with 21 targets, and a series of measurable indicators for each target, many of which are focused on health in specific target demographic groups, mainly children and pregnant women [1]. The initiation of these indicators, as well as a general growth in the number of health metric studies, has prompted substantial growth in approaches to measure them, with increasingly sophisticated methods that attempt to capture spatial heterogeneities in health conditions being developed (e.g. [2-10]).

An improved understanding of the geographic variation in health status and risks and access to services and care

\section{Biomed Central}


within countries is increasingly being recognized as central to meeting health and development goals and delivering equity in interventions and impacts [11-13]. For instance, approaches based on local epidemiological and coverage data have been identified as vital to achieving high impacts in reducing childhood mortality for MDGs 4 and 5 [14], while the subnational heterogeneity in HIV $[15,16]$ and malaria [5,17] prevalences mean that effective targeting of interventions remains vital in achieving MDG 6 [1]. Indicators assessed at national scales can often conceal important inequities, with the rural poor often least well represented [12,18]. Moreover, as international funding for health and development comes under pressure, the ability to target limited resources to underserved groups becomes crucial. Substantial demographic variations exist across countries and between urban and rural areas [19]. With MDG health indicators tied to specific vulnerable groups, there is a need to know where these vulnerable groups are and the number of individuals at risk that exist in order to accurately characterize denominators.

Health metrics continue to be collected, analyzed, and reported at national scales (e.g.[20-22]); however, datasets collected at subnational levels are increasingly available, and approaches that attempt to capture the spatial heterogeneity that often exists subnationally are being developed. The importance of geography is being recognized in development [23], mortality [24], and disease risks [19,25], with methods for mapping these factors at fine subnational scales becoming increasingly sophisticated and common in large-scale health metric studies [25]. While such projects are utilizing contemporary and fine resolution datasets to build the most spatially accurate evidence bases for MDG progress tracking, each are generally combined with spatial population datasets that contain no subnational information on target demographic groups to obtain denominators [19]. This lack of spatial data to quantify age groups by sex has meant that the increasing number of studies that are mapping indicators and risks subnationally continue to rely on simple national adjustments of spatial population data to provide denominators. For example, to estimate the number of children under 5 years old living at risk of $P$. falciparum malaria in Tanzania, previous work [5] has involved the development of a detailed map of prevalence from hundreds of community prevalence surveys, then overlaying this onto a detailed gridded population distribution dataset [26] to estimate total populations at risk, but then simply using the United Nations national-level estimate [27] of the proportion of the population that is under $5(17.9 \%)$ to convert this to an estimate of under-5s at risk, despite clear evidence of large subnational differences in the proportions of residential populations that are under 5 [19]. Further examples where similar national-level adjustments have been made include the estimation of numbers of pediatric fevers associated with malaria [28], numbers of preschool children at risk of anemia [10], schistosomiasis prevalence in children and under-20-year-olds [9,29], numbers of children residing in areas suitable for seasonal malaria chemoprevention [30], and global malaria mortality [6]. Moreover, in each of these cases, and for many other cartography-based health metric projects, the spatial demographic data used has been adjusted to a year of interest using national-level growth rates, ignoring the fact that the population distribution of a country changes heterogeneously over time [19].

Here we assess the importance of accounting for subnational demographic variations in deriving health metrics. We present the development of $\sim 100 \mathrm{~m}$ spatial resolution age- and sex-structured spatial population datasets for Africa built from satellite imagery and over a million measurements derived from more than 20,000 subnational administrative units and originating from a variety of publicly available sources that include census data and national household surveys. The effects of accounting for subnational demographic heterogeneity on estimates of the numbers of women of childbearing age and children under 5 years old impacted by long travel times to services and risks of malaria transmission, respectively, are then quantified to provide illustrative analyses.

\section{Methods}

\section{Constructing a detailed and contemporary population distribution dataset}

The AfriPop project (www.afripop.org) has recently completed construction of 2010 and 2015 estimates of population distribution for continental Africa and Madagascar at approximately $100 \mathrm{~m}$ spatial resolution. Full details are provided in Linard et al. [31] and on the project website (www.afripop.org). Briefly, a GIS-linked database of census and official population estimate data was constructed, targeting the most recent and spatially detailed datasets available, given their importance in producing accurate mapping [31-33]. Detailed maps of settlement extents were derived from Landsat satellite imagery through either semi-automated classification approaches [33,34] or expert opinion-based analyses [31]. These settlement maps were then used to refine land cover data, while local census data mapped at fine resolution enumeration area level from sample countries across the continent were exploited to identify typical regional per-land cover class population densities, which were then applied to redistribute census counts to map human population distributions at $100 \mathrm{~m}$ spatial resolution continent-wide [31,33,35]. Where available, additional country-specific datasets providing valuable data on population distributions not captured by censuses, such as internally displaced people or detailed national surveys, were incorporated into the mapping process [36]. 


\section{Compiling national estimates of age and sex structures}

In order to examine the effects of utilizing subnational data on age and sex structures of populations, 2010 national-level data were first obtained to provide a baseline for comparison. These were obtained from the United Nations Population Division's World Population Prospects 2010 publication [27] and are derived from national-level demographic models built upon census data. These national-level proportions were then used to adjust the gridded population dataset described above to produce separate five-year age group gridded datasets by sex, following approaches used in many previous studies that assume demographic homogeneity across countries (e.g. $[6,9,10,28,30,37])$.

\section{Compiling subnational estimates of age and sex structures}

Data on subnational population compositions from the last 20 years were obtained from a variety of sources for all mainland African countries, plus Madagascar (Additional file 1: Protocol S1). Contemporary censusbased counts broken down at a fine resolution administrative unit level generally provide the most reliable source for population composition mapping, due to the large sample sizes providing reliable information summarized for small areas. Where age and sex data reported at subnational levels were available for censuses undertaken within the last two decades, these were obtained for this study (Additional file 1: Protocol S1). An addition to the aggregated full census data are large samples of household-level records derived from censuses (census microdata) that provide age and sex structure, reported generally by administrative level 1 (e.g., province) or 2 (e.g., district). Census microdata on subnational age and sex proportions by subnational regions for African countries within the last twenty years were obtained where available (Additional file 1: Protocol S1). While census data are often readily available for high-income countries, for African countries census data with subnational reporting of age and sex structure can often be either unavailable or substantially more than a decade old. Alternative national household survey data sources were therefore exploited to provide the most contemporary and spatially detailed estimates as possible of age and sex proportions, given the constraints of their sampling frameworks. Here, national household survey data on population age and sex compositions were obtained from the most recent Demographic and Health Survey (DHS), Malaria Indicator Survey (MIS), or AIDS Indicator Survey (AIS) [38], or from Multiple Indicator Cluster Surveys (MICS) [39], for all countries where such surveys have been undertaken.

Summaries of subnational population structure by sex and five-year age groupings from either full national census summaries, census microdata, or household surveys were obtained for 47 of the 50 countries in mainland Africa, plus Madagascar. Where multiple datasets from similar time periods were available, the census or census microdata were given priority for use, due to the larger sample sizes. For four countries (Libya, Eritrea, Western Sahara, and Equatorial Guinea), no subnational estimates of age and sex structures were found, and for these countries the UN national estimates and projections for the 2000-2015 period [27] were obtained and used in the mapping. The relatively small sample sizes for household survey data and census microdata compared to those from full census data mean that age and sex proportions derived from them are more uncertain. To ensure that age proportions derived from these datasets were representative of those derived from census data, instances where (i) national household surveys were undertaken in the same year or within one year of a national census and (ii) census microdata samples and the full census that each was derived from were collated and statistical comparisons undertaken, which showed consistent and strong correlations (Additional file 1: Protocol S1).

Once datasets on numbers and proportions of individuals by age and sex had been collated for as many subnational units as available within the last two decades, using sample weights where applicable to household surveys, these were matched to corresponding GIS datasets showing the boundaries of each unit. Africa-wide GISlinked data on proportions of individuals by age and sex and by administrative unit were created for as close to 2010 as was available (Figure 1, further datasets are provided in Additional file 1: Protocol S1).

\section{Subnational and urban growth rates, projections, and adjustments}

The production of spatial population datasets for Africa has previously relied on simple interpolation between census-derived timepoints where available or, more commonly, the application of UN Population Division national-level growth rate estimates [27]. For 45 of the 50 countries in mainland Africa plus Madagascar, subnational growth rates derived from either censuses or official national estimates were obtained (see Additional file 1: Protocol S1 for details). Additionally, separate growth rates for urban and rural areas nationally were obtained for those countries and time periods for which subnational growth rate data were not available [40]. Finally, estimated population sizes for named African cities [40], and the urban extents dataset used in the construction of the Global Rural Urban Mapping Project (GRUMP) [26] were obtained. The urban extents matching those African cities for which individual population totals are estimated in the 

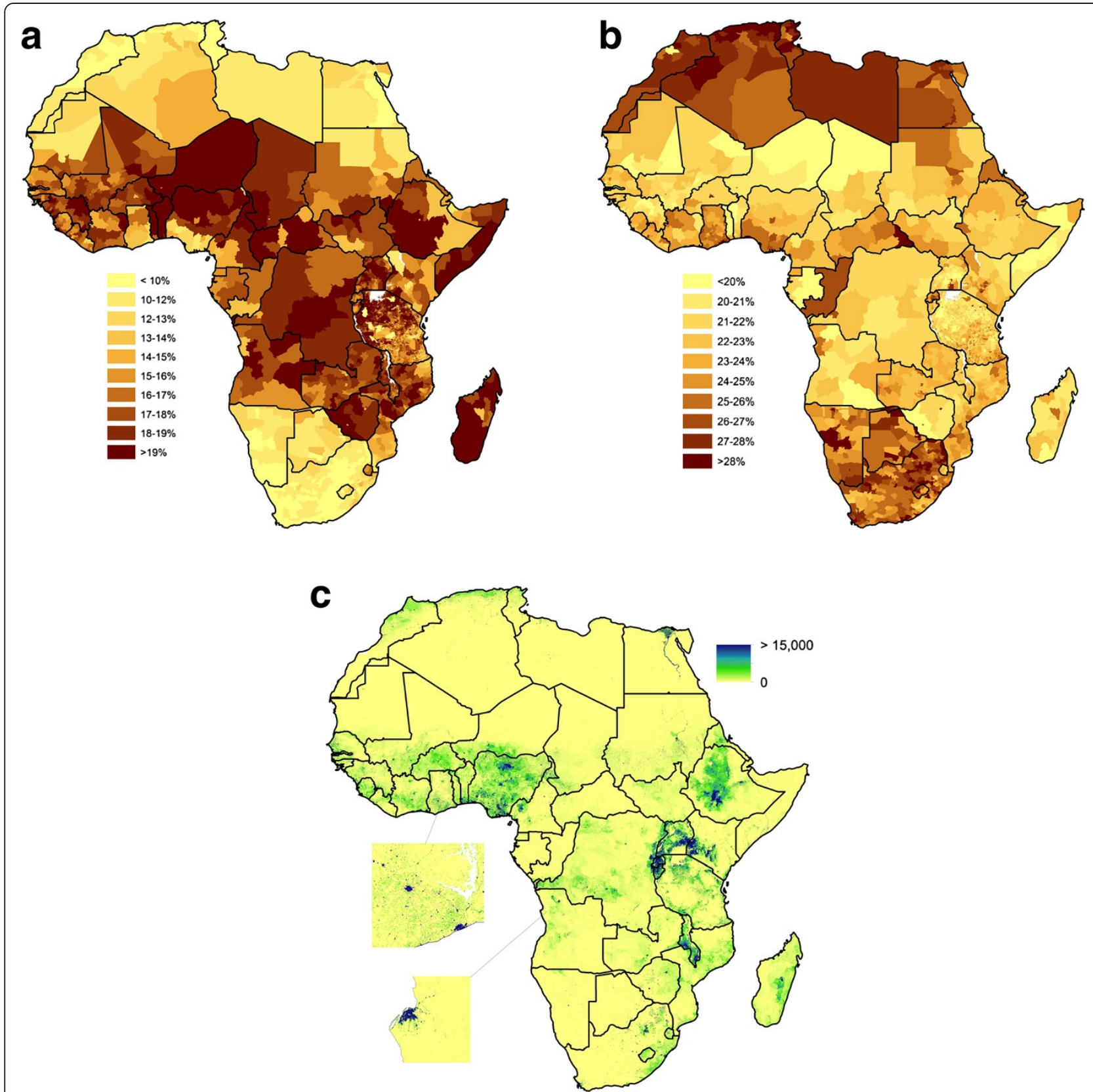

Figure 1 Spatial demographic datasets for mainland Africa and Madagascar. (a) The estimated proportion of children under 5 years old subnationally; (b) the estimated proportion of women of childbearing age subnationally; (c) the Africa-wide $1 \mathrm{~km}$ spatial resolution gridded dataset of numbers of children under 5 years old in 2010, with close-ups showing 100m spatial resolution detail for southern Ghana and Luanda, Angola.

UN World Urbanization Prospects [40] were identified, and the totals for 2000-2015 matched up.

\section{Gridded population dataset production}

The GIS unit-linked age and sex subnational proportions dataset described above was used to adjust the existing AfriPop 2010 spatial population datasets [31], to produce estimates of the distributions of populations by sex and five-year age group across Africa in 2010. The datasets were then adjusted to ensure that national population totals by age group, specific city totals and urban/ rural totals matched those reported by the UN $[27,40]$. For the analyses outlined in the remainder of this paper, the summation of the datasets representing males and females in the 0-5 year age group was undertaken to produce a 2010 distribution dataset of children under 5 years old, and the summation of datasets representing females in the $15-49$ year age groups was undertaken to produce a 2010 
dataset of women of childbearing age. The application of subnational growth rates to produce 2000, 2005, and 2015 datasets is described in Additional file 1: Protocol S1.

\section{Quantifying effects of spatial population dataset on health metrics}

To examine the effects on health and development indicators through use of the new subnational characterizations of children under 5 and women of childbearing age compared to undertaking national-level age adjustments using the UN data [27], two sets of illustrative analyses were undertaken. Firstly, Africa-wide estimates of the number of children under 5 years old residing in different Plasmodium falciparum malaria prevalence classes were calculated, and secondly, estimates of the number of women of childbearing age residing at different travel times from the nearest major settlement (population >50,000) across Africa and nearest health facility for countries with open access geolocated datasets of facilities were estimated. In each case the focus was on the size of the change in output metrics through accounting for demographic spatial heterogeneity, rather than the estimates produced and their fidelity.

One component of MDG 6 is an aim to halt and begin to reverse the incidence of malaria [1], with targets focused on those under 5 years of age, upon whom the greatest burden from the disease falls. To assess achievement of these targets, and the derivation of malaria metrics in general, maps of malaria prevalence are increasingly being used in combination with spatial population datasets to estimate numbers at risk and burdens (e.g.[5,6,41,42]). The Malaria Atlas Project (www.map.ox.ac.uk) has recently published a mapped distribution of the intensity of $P$. falciparum transmission in 2010 based upon infection prevalence among children aged 2 to 10 years (PfPR2-10) [5]. Here, the estimated distribution of prevalence by classes that have been proposed in the selection of suites of interventions at scale to reach control targets at different time periods [43,44] (Figure 2a) was used to extract estimated numbers of children under 5 years old per country residing in these different prevalence classes from (i) the AfriPop 2010 population dataset [31] adjusted to represent children under 5 using UN national proportion estimates [27] as described above, and (ii) the dataset of the 2010 population under 5 constructed from subnational data described above. For both datasets, national population totals were adjusted to match UN reported numbers [27] to ensure that any differences seen in numbers at risk were due solely to the addition of subnational information on under-5 proportions. Further details are provided in Additional file 2: Protocol S2.

Improving access to and for remote populations is an important priority for many of the MDG targets, such as those focused on eradicating extreme poverty, achieving universal primary education, and developing a global partnership for development [1]. Moreover, each healthrelated goal is dependent upon accessing populations to provide interventions, principally delivered through health facilities, and the difficulty in traveling to these facilities has been consistently highlighted as a barrier to treatment in rural populations, particularly in maternal health $[45,46]$. The measurement of accessibility or "remoteness" of populations is therefore of importance in measuring progress toward achieving these goals, and increasingly, approaches based on GIS-derived travel times are being applied [45-51]. A recently developed continent-wide travel time dataset $[52,53]$ was used here to map those regions estimated to be greater than five hours from the nearest settlement of population size greater than 50,000. This dataset was used as an illustrative proxy for health system access, since reliable continent-wide datasets on health facility locations do not currently exist. To demonstrate the size of the variations achieved when using actual health facility data, for eight countries with open-access datasets of health facility locations (Additional file 2: Protocol S2), maps representing estimated travel times to the nearest facilities were constructed following previous approaches [50-55] (Additional file 2: Protocol S2). The accessibility datasets were used to extract estimated numbers of women of childbearing age per country residing in different travel time classes from (i) the AfriPop 2010 population dataset [31] adjusted to represent women of childbearing age using UN national proportion estimates [27] as described above and (ii) the 2010 distribution dataset of women of childbearing age constructed from subnational data described above.

\section{Results}

\section{Data assembly and risk group distributions}

Over a million individual data records were collected and matched to 20,381 administrative units across the continent (Figure 1a, Additional file 1: Protocol S1). The subnational proportions (Figures 1a, 1b) and growth rates (Additional file 1: Protocol S1) were combined with existing spatial population datasets [31] and UN country total estimates [27] as described above to produce highresolution age-and sex-structured Africa-wide spatial population datasets for 2000, 2005, 2010, and 2015 (Figure 1c, Additional file 1: Protocol S1). For comparison of the effects of the inclusion of subnational age and sex structure data on health metrics, the same spatial population count dataset [31] was combined with UN Population Prospects national-level data on age and sex proportions [27], as described above, to produce alternative high-resolution Africa-wide spatial population datasets that assumed homogeneity in age and sex structures across countries. 
The distributions of the proportion of children under 5 at subnational scales (Figure 1a) and women of childbearing age (Figure 1b), show the great differences that exist on a continental scale between the majority of subSaharan African countries and those higher-income countries in southern and northern Africa. Moreover, large variations are also seen within country borders, whether the proportions are measured at the coarsest subnational units of administrative level 1 (provincial) or as fine a detail as level 4 (wards in Tanzania). This great heterogeneity in subnational population composition across the continent is often ignored in the application of existing spatial population datasets, thus assuming demographic homogeneity across countries (e.g. [6,9,10,28,30,37]).

An indication of the size of subnational variation in the proportion of the population under 5 captured in the subnational level dataset (Figure 1a) that is missed through summarization to national levels is highlighted in Figure 3. The size of this variation is related to the administrative unit level of the input data, with those
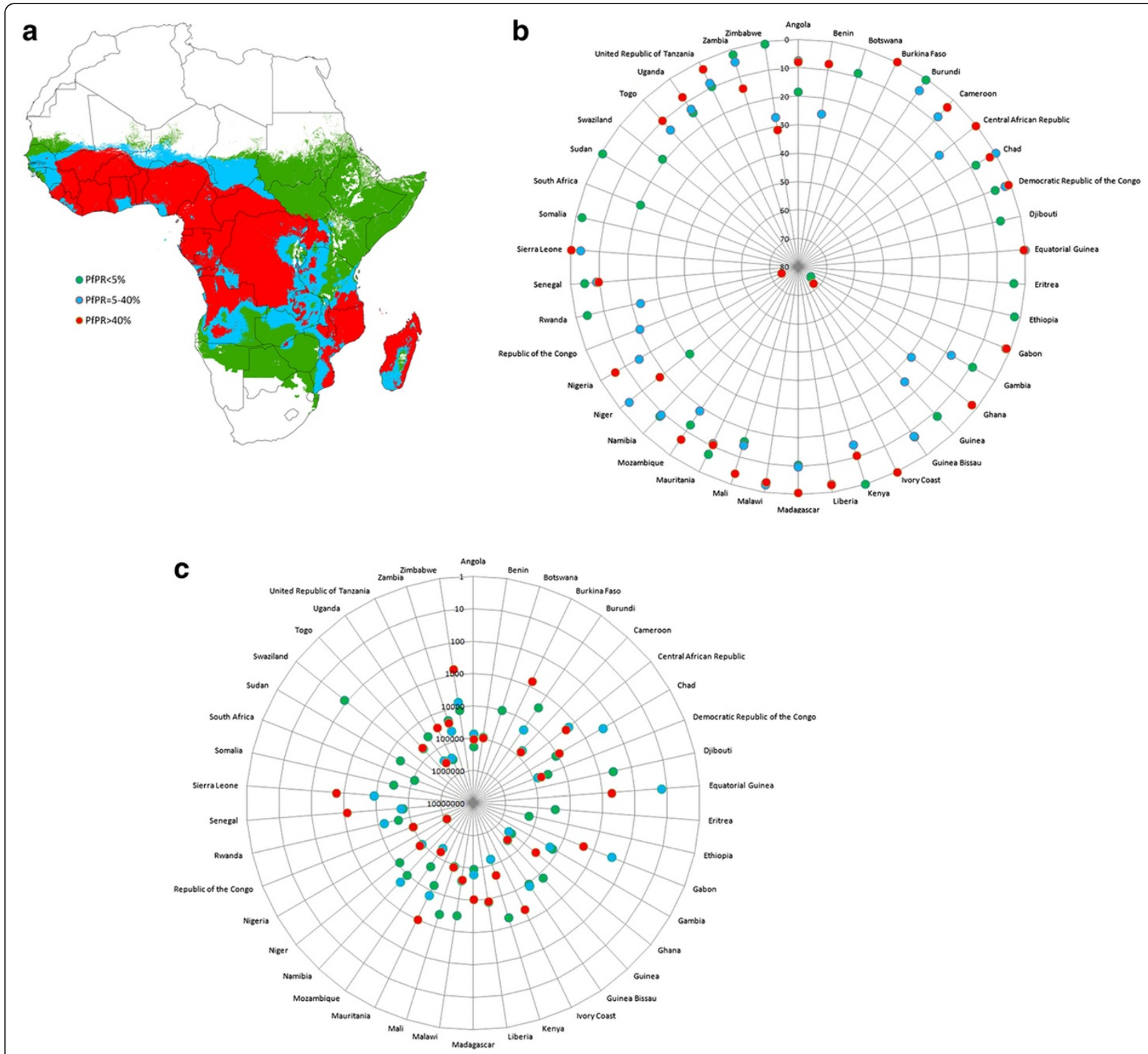

Figure 2 P. falciparum malaria prevalence in Africa and the effects on metrics of accounting for subnational age structure. (a) Predicted prevalence classes for $P$. falciparum malaria in Africa [5]. (b) The absolute percentage changes in estimated numbers of children under 5 years old residing under the three prevalence classes through changing from using UN national proportions [27] to produce per grid cell estimates of numbers under 5 years to using the subnational proportion data assembled here (Additional file 1: Protocol S1). (c) The changes in estimated numbers of children under 5 years old residing under the three prevalence classes through changing from using UN national proportions [27] to produce per grid cell estimates of numbers under 5 years to using the subnational proportion data assembled here (Additional file 1: Protocol S1). In (b) and (c), data values are only plotted when a transmission class encompasses $>10 \%$ of the population of a country. 


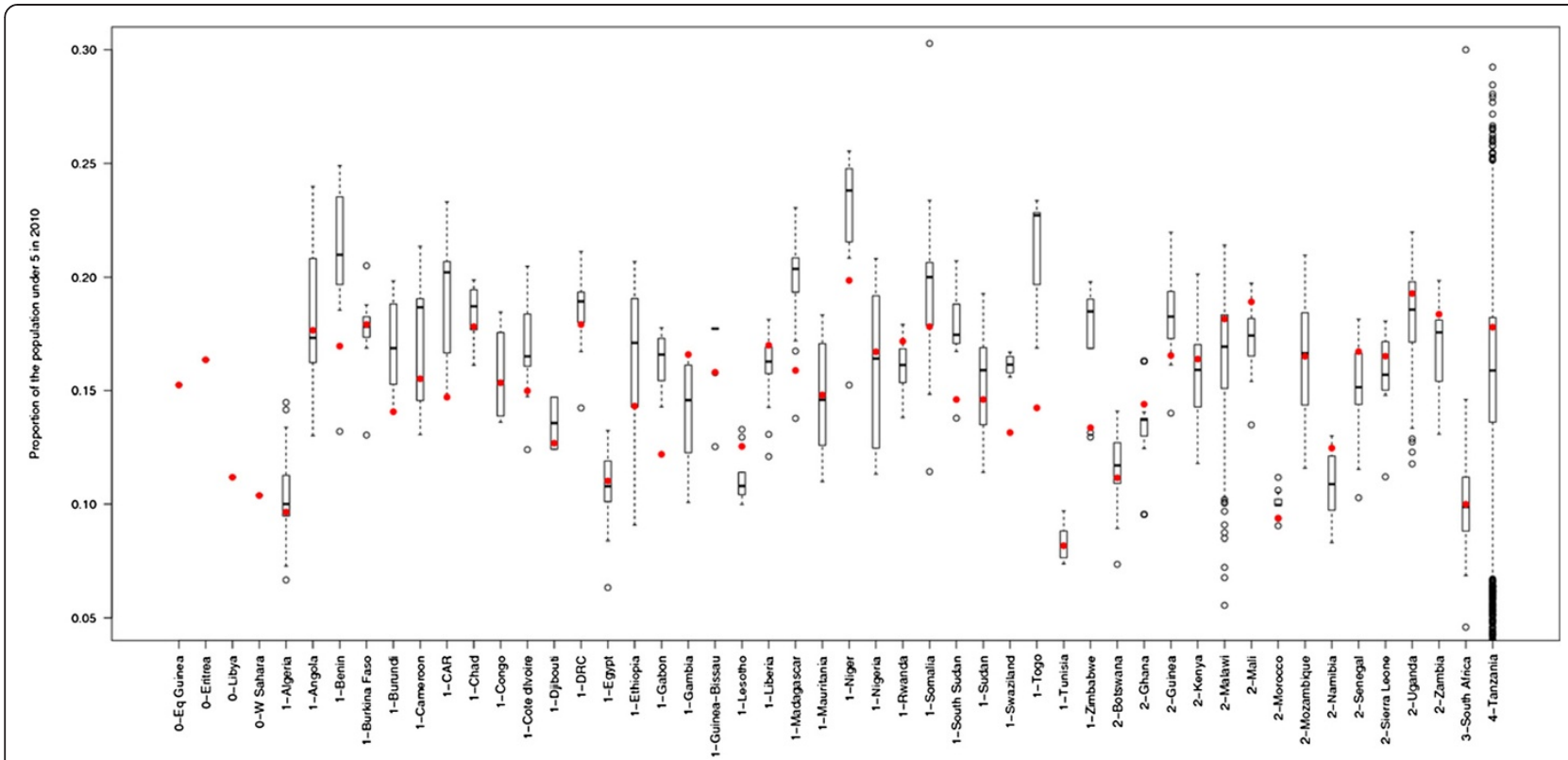

Figure 3 Differences between national and subnational summaries of population proportions under 5 years old for African countries. Comparison of UN national estimates [27] of proportions of the population under 5 years old in 2010 (red dots) against the range of proportions measured by the subnational datasets collated here (Additional file 1: Protocol S1) shown as boxplots. The solid center line of the boxplot shows the median values, the box width represents the interquartile range, and the whiskers extend to 1.5 times the interquartile range from the box (values further away than this are shown as open circles). The administrative unit level of the subnational data used here is shown as a prefix to the country name on the $x$-axis.

countries for which the most spatially detailed age structure data were available (Tanzania and South Africa) showing the largest differences between minimum and maximum estimates of per-unit proportions of residents under 5. Nevertheless, even for those countries where subnational data were only available at administrative unit level 1, differences of $+/-5 \%$ from the UN national estimates are common. Moreover, national estimates are often reflective of proportions in urban areas, where the majority of people reside, hiding the extremes that exist in rural areas, and this is evidenced by the UN estimates falling outside of the interquartile range of the boxplots in many cases (Figure 3).

\section{Effects on health metrics}

In terms of using cartographic approaches to estimating the numbers of people in specific age groups either impacted by disease or able to access large settlements and health facilities, it is clear that the use of subnational data on age structures can result in substantial differences in output indicators over simply using nationallevel proportions. Figure 2 shows these differences for each country in estimates of the number of children under 5 residing under different $P$. falciparum malaria prevalence classes. The simple use of national level age structure adjustments for estimates of numbers within different age groups at risk of $P$. falciparum malaria, as undertaken in many recent studies, can result in some estimates being nearly $100 \%$ different from those obtained using more detailed data that capture subnational demographic variations, with close to half of countries exhibiting absolute differences greater than 10\% (Figure 2b). For small countries, such as Guinea-Bissau, this translates to estimates changing by only a few thousand people, whereas for more populous countries, such as Nigeria, this results in many millions of children changing classes (Figure 2c). Such trends are also seen when quantifying travel times to major settlements and health facilities for women of childbearing age (Figure 4). At a continental scale, estimates of numbers residing more than five hours from a large settlement are greater than $10 \%$ different for half of the countries if an assumption of age structure homogeneity across the country is applied, compared to accounting for the subnational variations that exist (Figure 4a). Further, similar findings are evident when estimating numbers of women of childbearing age residing at different travel times from health facilities (Figure $4 \mathrm{~b}$ ), emphasizing the impact that spatial demographic heterogeneity has upon the measurement of health facility access for vulnerable populations. For six of the eight countries examined, if the proportion of the population that women of childbearing age make up is assumed to match national estimates [27] across the country, then the numbers residing at travel times of more than an hour from the nearest health facility will be consistently underestimated (Figure 4b) due to subnational variations in age and sex structures. For 


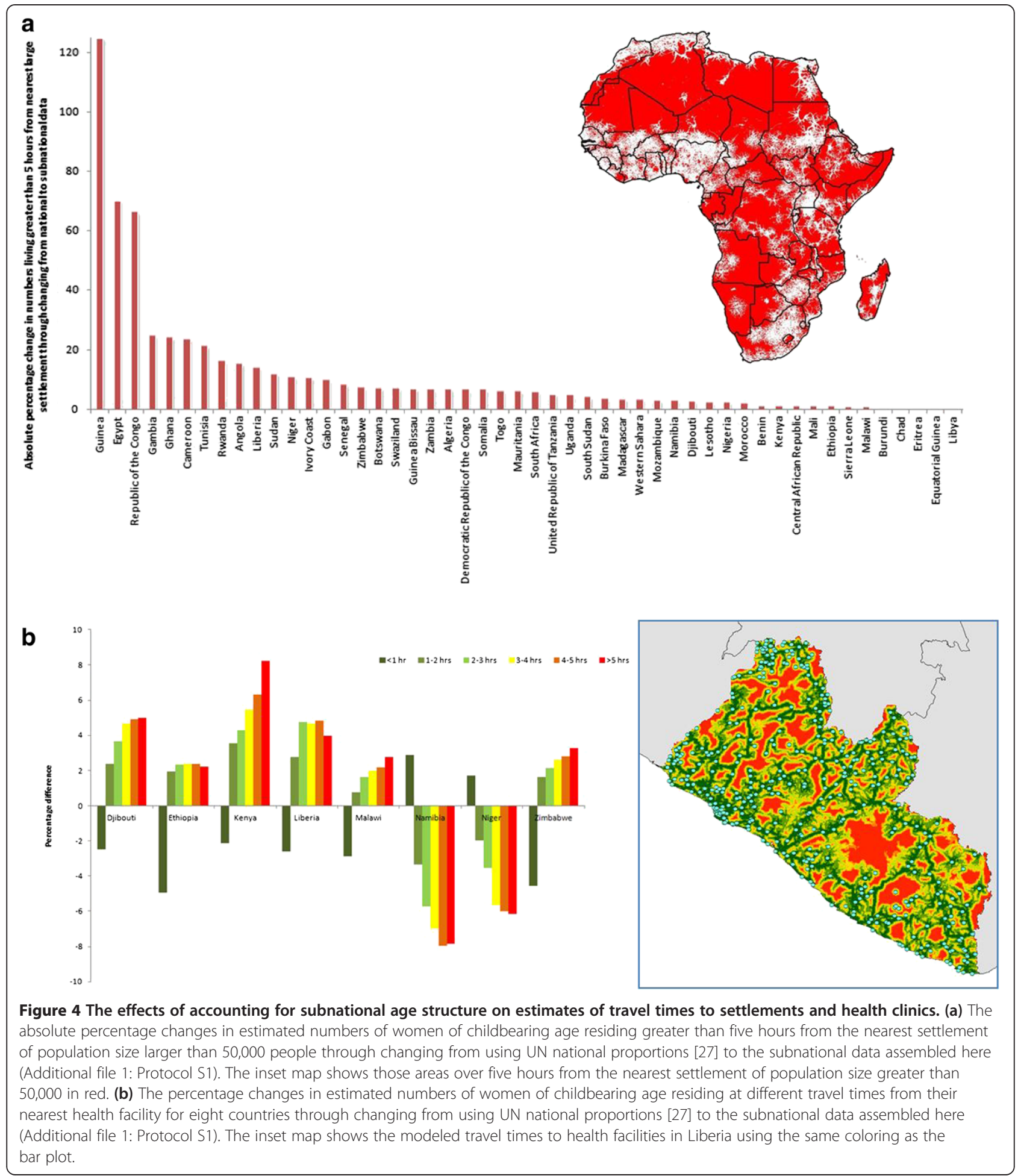

Namibia and Niger, the two most sparsely populated countries, the reverse is true.

\section{Discussion}

The assessment of progress toward meeting the MDGs will be measured through national-level indicators [1] that can mask substantial inequalities across nations $[12,18,56]$. The development of cartographic approaches to transforming georeferenced data on health and development metrics into valuable spatial datasets is opening opportunities for quantitative assessments of these inequalities, the targeting of interventions and measurement of progress toward the 
MDGs, but demographic spatial datasets to support such efforts remain reliant on coarse and outdated input data for accurately locating risk groups.

While high-resolution spatial data on population distributions in resource poor areas are now becoming available (e.g., www.afripop.org, www.asiapop.org, www.census.gov/ population/international/data/mapping/demobase.html), comprehensive and contemporary subnational information on the demographic attributes of these populations remain scattered across national statistics office reports and household surveys [19]. Here approaches to combining these publicly available disparate datasets are presented, enabling the production of Africa-wide datasets depicting age and sex compositions at subnational scales. The datasets and analyses highlight the importance of accounting for subnational demographic variations in deriving health and developments metrics. Both the large subnational variations in age and sex population structures that are evident (Figures 1 and 3), and the resulting impacts that these have on metric derivation (Figures 2 and 4) underline the need to obtain and utilize the most spatially refined data available.

The ranges of proportions of the population that is under 5 years old seen when comparing the subnational versus national-level estimates (Figure 3) highlight the need for more spatially detailed demographic data to better capture these variations. Differences of $+/-5 \%$ in the proportions are common, and the spatial configuration of those areas that are substantially greater or less than the UN estimates in relation to the spatial distribution of disease risks or access, as seen in Figures 2 and 4 , can have major implications on the derivation of indicators. Whilst the distributions of predicted malaria risk or travel times are mapped as continuous variables at $1 \mathrm{~km}$ spatial resolution, if the population distribution data used to derive numbers at risk is based upon an assumption of age and sex structure homogeneity through national-level estimates, it is clear that this can result in some significant inaccuracies that consistently remain unacknowledged. Clear urban and rural differences (Additional file 1: Protocol S1) also highlight the need for accounting for such variations, and when indicators such as malaria risk or access to health facilities that vary substantially by urban-rural divides are being estimated, the large effects of this are evident (Figures 2 and 4). For example, in Kenya some of the most rural areas have the highest malaria transmission, the largest travel times to health facilities, and the highest proportions of children under 5/lowest proportions of women of childbearing age. Thus, accounting for all three of these factors subnationally compared to assuming a homogenous demographic structure results in substantial differences in outcome metrics (Figures 2 and 4). As funding for health and poverty-related mapping and the number of new cartography projects (e.g. [57-60]) continues to grow, the need for accurate spatial population distribution data will also grow if denominator-reliant metrics are required.

While accounting for subnational heterogeneity in population attributes likely results in significant improvements in the accuracy of health metrics, it is clear that many sources of uncertainty and error remain. All of the census and survey-based data used here are subject to various sources of error and bias, many of which have been well documented [61]. Indigenous groups, informal settlements, places experiencing civil unrest, and refugees are often entirely unsampled, either because of political biases, missing sampling frames, or prohibitive difficulties in carrying out a survey. Uncertainties also arise over comparisons being made between primarily census-based national estimates of age/sex proportions from the UN Population Prospects [27] and the household surveyderived subnational age/sex proportions used here for some countries. Differences between the way these proportions were measured contributes to uncertainties in comparisons between outcome health metrics, though strong correlations between the household survey-derived age structures and those derived from census data suggest that such differences may be small (Additional file 1: Protocol S1). Further, the underlying AfriPop population datasets contain uncertainties [31], while for some countries, the input data used here remains outdated and coarse (Figure 1, Additional file 1: Protocol S1). Like most other population parameters reported for administrative polygons, the age and sex proportions are also subject to the modifiable areal unit problem [62]. Discretising (by gridding) a phenomenon that is continuous (or in this case, varying at a far higher resolution) is an arbitrary process. In the case of the datasets presented here, whilst the precision with which heterogeneities in vulnerable population distributions are mapped is improved over simple national adjustments, we are still faced with a dataset containing one set of values for Libya and thousands for Tanzania. There is therefore a need to more rigorously quantify the uncertainties inherent in spatial demographic datasets. The advancement of theory, increasing availability of computation, and growing recognition of the importance of robust handling of uncertainty have all contributed to the emergence in recent years of new, sophisticated Bayesian approaches to the large-scale modeling and mapping of disease $[4,7,25]$, but such methods have yet to cross over to the spatial demographic databases with which such maps are used. The regular availability of new national household surveys means that more contemporary data is continually becoming available to aid in updating and improving the accuracy of the datasets presented here, potentially through automated systems that can rapidly adapt to new incoming data and 
integrate them into the output spatial datasets, alongside robust methods to account for temporal differences [63].

The international focus on health-related goals coupled with a growing trend in research and funding for cartographic approaches to deriving metrics are increasing needs for spatial demographic data of similar scope for use in estimating denominator sizes and characteristics of populations at risk. The importance of accounting for subnational demographic variations in deriving health metrics is clear and the size of the differences that exist between ignoring subnational variations in age and sex structures, compared to accounting for them, is large enough to make the difference between success and failure in meeting a MDG. Here we have shown that sufficient data exists to produce a continent-wide subnational picture of demographic attributes and the mapping of key risk group distributions. Gridded age-structured datasets for 2000, 2005, 2010, and 2015 are freely available to download from the AfriPop project website (www.afripop.org) and will be regularly updated as new data become available. Similar datasets for Asia and Latin America will soon be made available through the AsiaPop (www.asiapop.org) and AmeriPop (www.ameripop.org) projects.

\section{Additional files}

Additional file 1: Protocol S1. Constructing spatial demographic datasets for Africa.

Additional file 2: Protocol S2. Calculating cartographically-derived health metrics.

\section{Competing interests}

The authors have declared that no competing interests exist.

\section{Authors' contributions}

AJT conceived the study, undertook data assembly and analyses, and wrote the paper. AJG, AEG, MG, and $C L$ aided with data analyses and writing the paper. PWS and AMN aided with data collection, processing, and writing the paper. All authors read and approved the final manuscript.

\section{Acknowledgments}

The authors thank Sakshi Tikya, Peng Jia, Nick Campiz, Viola Kurui, Victor Alegana, and Betsy Makena for help with data assembly and Drs Simon Hay and Peter Gething for providing the global P. falciparum dataset. This paper forms part of the output of the Afripop population mapping project (www. afripop.org), and a full list of acknowledgements of those who provided data can be found on the website. AJT acknowledges funding support from the RAPIDD program of the Science and Technology Directorate, Department of Homeland Security, and the Fogarty International Center, National Institutes of Health, and is also supported by grants from NIH/NIAID (U19AI089674) and the Bill \& Melinda Gates Foundation (\#49446 and \#1032350), which support AEG. RWS is supported by the Wellcome Trust as Principal Research Fellow (\#079080). AMN is supported by a Wellcome Trust Intermediate Research Fellowship (\#\#095127). Both RWS and AMN are also supported by Wellcome Trust Major Overseas Programme grant to the KEMRI/Wellcome Trust Research Programme (\#092654). AJG is partially supported by the National Science Foundation (Grant \#0801544) in the Quantitative Spatial Ecology, Evolution and Environment Program at the University of Florida. CL is supported by a fellowship from the Fonds National de la Recherche Scientifique (FRS/FNRS), Belgium. The funders had no role in study design, data collection and analysis, decision to publish, or preparation of the manuscript.

\section{Author details}

'Department of Geography and Environment, University of Southampton, Highfield, Southampton, UK. '2Fogarty International Center, National Institutes of Health, Bethesda, MD 20892, USA. ${ }^{3}$ Department of Geography, University of Florida, Gainesville, Florida, USA. ${ }^{4}$ Emerging Pathogens Institute, University of Florida, Gainesville, Florida, USA. ${ }^{5}$ Malaria Public Health and Epidemiology Group, Centre for Geographic Medicine, KEMRI - Univ. Oxford - Wellcome Trust Collaborative Programme, Nairobi, Kenya. ${ }^{6}$ Centre for Tropical Medicine, Nuffield Department of Clinical Medicine, University of Oxford, CCVTM, Oxford, UK. ${ }^{7}$ Biological Control and Spatial Ecology, Université Libre de Bruxelles, Brussels, Belgium. ${ }^{8}$ Fonds National de la Recherche Scientifique (F.R.S./FNRS), Brussels, Belgium.

Received: 24 October 2012 Accepted: 11 July 2013

Published: 23 July 2013

\section{References}

1. United Nations Millenium Declaration. http://www.un.org/millennium/ declaration/ares552e.pdf.

2. Brooker S: Spatial epidemiology of human schistosomiasis in Africa: risk models, transmission dynamics and control. Trans $R$ Soc Trop Med Hyg 2007, 101:1-8

3. Brooker S, Hotez PJ, Bundy DA: The global atlas of helminth infection: mapping the way forward in neglected tropical disease control. PLOS Negl Trop Dis 2010, 4:e779.

4. Gething PW, Patil AP, Hay SI: Quantifying aggregated uncertainty in Plasmodium falciparum malaria prevalence and populations at risk via efficient space-time geostatistical joint simulation. PLOS Com Biol 2010, 6:e1000724.

5. Gething PW, Patil AP, Smith DL, Guerra CA, Elyazar IR, Johnston GL, Tatem AJ, Hay SI: A new world malaria map: Plasmodium falciparum endemicity in 2010. Malar J 2011, 10:378.

6. Murray CJ, Rosenfeld LC, Lim SS, Andrews KG, Foreman KJ, Haring D, Fullman N, Naghavi M, Lozano R, Lopez AD: Global malaria mortality between 1980 and 2010: a systematic analysis. Lancet 2012, 379:413-431.

7. Patil AP, Gething PW, Piel FB, Hay SI: Bayesian geostatistics in health cartography: the perspective of malaria. Trends Parasitol 2011, 27:246-253.

8. Riedel N, Vounatsou P, Miller JM, Gosoniu L, Chizema-Kawesha E, Mukonka $\checkmark$, Steketee RW: Geographical patterns and predictors of malaria risk in Zambia: Bayesian geostatistical modelling of the 2006 Zambia national malaria indicator survey (ZMIS). Malar J 2010, 9:37.

9. Schur N, Hurlimann E, Garba A, Traore MS, Ndir O, Ratard RC, Tchuente LT, Kristensen TK, Utzinger J, Vounatsou P: Geostatistical model-based estimates of schistosomiasis prevalence among individuals aged $<20$ years in West Africa. PLoS Negl Trop Dis 2011, 5:e1194.

10. Soares Magalhaes RJ, Clements ACA: Mapping the risk of anaemia in preschool-age children: the contribution of malnutrition, malaria and helminth infections in West Africa. PLoS Med 2011, 8:e1000438.

11. Global forum on local development: Localizing the MDGs. Kampala, Uganda: Global forum on local development; 2010.

12. Reidpath DD, Morel CM, Mecaskey JW, Allotey P: The Millennium Development Goals fail poor children: the case for equity-adjusted measures. PloS Med 2009, 6:e1000062

13. Stuckler D, Basu S, McKee M: Drivers of inequality in Millennium Development Goal progress: a statistical analysis. PLoS Med 2010, 7:e1000241

14. Kinney MV, Kerber KJ, Black RE, Cohen B, Nkrumah F, Coovadia H, Nampala PM, Lawn JE, Axelson H, Bergh AM, et al: Sub-Saharan Africa's mothers, newborns, and children: where and why do they die? PLoS Med 2010, 7:e1000294

15. Kleinschmidt I, Pettifor A, Morris N, MacPhail C, Rees H: Geographic distribution of human immunodeficiency virus in South Africa. AmJTrop Med Hyg 2007, 77:1163-1169

16. UNAIDS: Global Report: UNAIDS report on the global AIDS epidemic. Geneva, Switzerland: UNAIDS; 2010.

17. Snow RW, Amratia P, Kabaria CW, Noor AM, Marsh K: The changing limits and incidence of malaria in Africa: 1939-2009. Adv Parasitol 2012, 78:169-262.

18. Gwatkin DR: How much would poor people gain from faster progress towards the Millennium Development Goals for health? Lancet 2005, 365:813-817. 
19. Tatem AJ, Adamo S, Bharti N, Burgert CR, Castro M, Dorelien A, Fink G, Linard C, Mendelsohn J, Montana L, et al: Mapping populations at risk: Improving spatial demographic data for infectious disease modeling and metric derivation. Pop Health Metrics 2012, 10:8.

20. World Health Organization: World Health Statistics. Geneva: WHO; 2011

21. Lopez AD, Mathers CD, Ezzati M, Jamison DT, Murray CJ: Global Burden of Disease and Risk Factors. New York: The World Bank and Oxford University Press; 2006.

22. UNICEF: The State of the World's Children 2012: Children in an Urban World. New York: UNICEF; 2012

23. The World Bank: World Development Report 2009: Reshaping Economic Geography. Washington DC: The International Bank for Reconstruction and Development / The World Bank; 2009.

24. Storeygard A, Balk D, Levy M, Deane G: The global distribution of infant mortality: A subnational spatial view. Pop Space Place 2008, 14:209-229.

25. Linard C, Tatem AJ: Large-scale spatial population databases in infectious disease research. Int J Health Geogr 2012, 11:7.

26. Balk DL, Deichmann U, Yetman G, Pozzi F, Hay SI, Nelson A: Determining global population distribution: methods, applications and data. Adv Parasitol 2006, 62:119-156.

27. United Nations Population Division: World population prospects, 2010 revision. New York: United Nations; 2010.

28. Gething PW, Kirui VC, Alegana VA, Okiro EA, Noor AM, Snow RW: Estimating the number of paediatric fevers associated with malaria infection presenting to Africa's public health sector in 2007. PLoS Med 2010, 7:e1000301.

29. Schur N, Vounatsou P, Utzinger J: Determining treatment needs at different spatial scales using geostatistical model-based risk estimates of schistosomiasis. PLoS Negl Trop Dis 2012, 6:e1773.

30. Cairns M, Roca-Feltrer A, Garske T, Wilson AL, Diallo D, Milligan PJ, Ghani AC, Greenwood BM: Estimating the potential public health impact of seasonal malaria chemoprevention in African children. Nat Commun 2012, 3:881

31. Linard C, Gilbert M, Snow RW, Noor AM, Tatem AJ: Population distribution, settlement patterns and accessibility across Africa in 2010. PLoS One 2012, 7:e31743.

32. Hay SI, Guerra CA, Tatem AJ, Atkinson PM, Snow RW: Urbanization, malaria transmission and disease burden in Africa. Nat Rev Microbio/ 2005, 3.81-90.

33. Tatem AJ, Noor AM, von Hagen C, di Gregorio A, Hay SI: High resolution population maps for low income nations: combining land cover and census in East Africa. PLoS One 2007, 2:e1298.

34. Tatem AJ, Noor AM, Hay SI: Defining approaches to settlement mapping for public health management in Kenya using medium spatial resolution satellite imagery. Rem Sens Env 2004, 93:42-52.

35. Linard C, Gilbert M, Tatem AJ: Assessing the use of global land cover data for guiding large area population distribution modelling. GeoJournal 2010, 76:525-538.

36. Linard C, Alegana VA, Noor AM, Snow RW, Tatem AJ: A high resolution spatial population database of Somalia for disease risk mapping. Int $J$ Health Geogr 2010, 9:45.

37. Noor AM, Mutheu JJ, Tatem AJ, Hay SI, Snow RW: Insecticide-treated net coverage in Africa: mapping progress in 2000-07. Lancet 2008, 373:58-67.

38. Demographic and Health Surveys. http://www.measuredhs.com/

39. Multiple Indicator Cluster Survey (MICS). http://www.unicef.org/statistics/ index 24302.html.

40. United Nations Population Division: World urbanization prospects, 2011 revision. New York: United Nations; 2011

41. Hay SI, Okiro EA, Gething PW, Patil AP, Tatem AJ, Guerra CA, Snow RW: Estimating the global clinical burden of Plasmodium falciparum malaria in 2007. PLoS Med 2010, 7:e100029.

42. World Health Organization: The World Malaria Report. Geneva, Switzerland: World Health Organization; 2011.

43. Hay SI, Smith DL, Snow RW: Measuring malaria endemicity from intense to interrupted transmission. Lancet Infect Dis 2008, 8:369-378.

44. Smith DL, Hay SI: Endemicity response timelines for Plasmodium falciparum elimination. Malar J 2009, 8:87.

45. Gabrysch S, Cousens S, Cox J, Campbell OM: The influence of distance and level of care on delivery place in rural Zambia: a study of linked national data in a geographic information system. PLoS Med 2011, 8:e1000394.

46. Gething PW, Johnson FA, Frempong-Ainguah F, Nyarko P, Baschieri A Aboagye P, Falkingham J, Matthews Z, Atkinson PM: Geographical access to care at birth in Ghana: a barrier to safe motherhood. Southampton, UK: Centre for Population Change; 2012

47. Apparicio P, Abdelmajid M, Riva M, Shearmur R: Comparing alternative approaches to measuring the geographical accessibility of urban health services: Distance types and aggregation-error issues. Int I Health Geogr 2008, 7:7.

48. Guagliardo MF: Spatial accessibility of primary care: concepts, methods and challenges. Int J Health Geogr 2004, 3:3.

49. Noor AM, Amin AA, Gething PW, Atkinson PM, Hay SI, Snow RW: Modelling distances travelled to government health services in Kenya. Trop Med Int Health 2006, 11:188-196.

50. Munoz UH, Kallestal C: Geographical accessibility and spatial coverage modeling of the primary health care network in the Western Province of Rwanda. Int J Health Geogr 2012, 11:40.

51. Modelling physical accessibility to health care and geographic coverage (AccessMod). http://www.who.int/kms/initiatives/accessmod/en/index.html.

52. Nelson A: Estimated travel time to the nearest city of 50,000 or more people in year 2000. Ispra, Italy: Global Environment Monitoring Unit, Joint Research Centre; 2008.

53. Uchida $H$, Nelson A: Agglomeration index: towards a new measure of urban concentration. In World Development Report 2009. New York: World Bank; 2009.

54. Alegana VA, Wright JA, Pentrina U, Noor AM, Snow RW, Atkinson PM: Spatial modelling of healthcare utilisation for treatment of fever in Namibia. Int J Health Geogr 2012, 11:6.

55. Blanford Jl, Kumar S, Luo W, Maceachren AM: It's a long, long walk: accessibility to hospitals, maternity and integrated health centers in Niger. Int J Health Geogr 2012, 11:24.

56. Rossouw S, Webber DJ: Millenium development goals: a concern over sub-national variations. Int Bus Econ Res J 2011, 10:127-135.

57. Smith JL, Haddad D, Polack S, Harding-Esch EM, Hooper PJ, Mabey DC, Solomon AW, Brooker S: Mapping the global distribution of trachoma: why an updated atlas is needed. PLoS Negl Trop Dis 2011, 5:e973.

58. Brady OJ, Gething PW, Bhatt S, Messina JP, Brownstein JS, Hoen AG, Moyes $\mathrm{CL}$, Farlow AW, Scott TW, Hay SI: Refining the global spatial limits of dengue virus transmission by evidence-based consensus. PLoS Negl Trop Dis 2012, 6:e1760.

59. Huang Z, Das A, Qiu Y, Tatem AJ: Web-based GIS: the vector-borne disease airline importation risk (VBD-AIR) tool. Int J Health Geogr 2012, 11:33.

60. Brooker S, Kabatereine NB, Smith JL, Mupfasoni D, Mwanje MT, Ndayishimiye O, Lwambo NJ, Mbotha D, Karanja P, Mwandawiro C, et al: An updated atlas of human helminth infections: the example of East Africa. Int J Health Geogr 2009, 8:42

61. Hobcraft J, McDonald J, Rutstein SO: Socioeconomic factors in infant and child mortality: a cross-national perspective. Pop Studies 1984, 38:193-223.

62. Openshaw S: The Modifiable Areal Unit Problem. Norwich: Geo Books; 1984.

63. Raftery AE, Li N, Sevcikova H: Gerland P. Heilig GK: Bayesian probabilistic population projections for all countries. Proc Natl Acad Sci USA; 2012.

doi:10.1186/1478-7954-11-11

Cite this article as: Tatem et al:: Millennium development health metrics: where do Africa's children and women of childbearing age live?. Population Health Metrics 2013 11:11.

\section{Submit your next manuscript to BioMed Central and take full advantage of:}

- Convenient online submission

- Thorough peer review

- No space constraints or color figure charges

- Immediate publication on acceptance

- Inclusion in PubMed, CAS, Scopus and Google Scholar

- Research which is freely available for redistribution 